\title{
Tamanho ótimo de parcela e número de repetições em soja (Glycine max (L.) Merril)
}

\author{
Optimum plot size and number of replications in soybeans (Glycine max (L.) Merril)
}

\author{
Thomas Newton Martin ${ }^{1}$ Luiz Marcelo Costa Dutra ${ }^{2}$ Adilson Jauer $^{3}$ \\ Lindolfo Storck ${ }^{4}$ Lucio Zabot $^{5}$ Daniel Uhry ${ }^{6}$ Antônio Luís Santi ${ }^{3}$ \\ Cassiano Stefanelo ${ }^{7}$ Orlando Antônio Lucca Filho ${ }^{8}$
}

\section{RESUMO}

\begin{abstract}
Com o objetivo de verificar se diferentes cultivares de soja alteram a estimativa do tamanho ótimo de parcela e do número de repetições, foi conduzido um experimento no ano agrícola de 2000/01 em área pertencente ao Departamento de Fitotecnia no Campus da Universidade Federal de Santa Maria. Utilizaram-se duas cultivares de soja (BRS 137 e Fepagro-RS 10), conduzidas no delineamento blocos ao acaso com três repetições. O tamanho de parcela foi estimado através do método da máxima curvatura modificada. Utilizou-se o teste de paralelismo de SEBER (1976), para verificar a heterogeneidade de resposta entre as cultivares testadas. O número de repetições foi estimado através da metodologia descrita por HATHEWAY (1961). Pode-se concluir que, para as cultivares estudadas, o número de repetições que confere uma precisão adequada é igual a sete e o tamanho ótimo de parcela é de $3,96 \mathrm{~m}^{2}$.
\end{abstract}

Palavras-chave: tamanho ótimo de parcela, número de repetições, heterogeneidade do solo e do material experimental.

\section{ABSTRACT}

With the objective of verifying if different cultivars of soybean modify the estimates of the optimum plot size and of the numbers of replications, an experiment was conduced in the agriculturist year of 2000/01 in area pertaining to the Department of Crop Science in Santa Maria University Campus. Two cultivars of soybean were used (BRS 137 and Fepagro-RS10), conducted in a randomized block design with three replications. The size of plot was evaluated through the method of maximum modified bending. The test of parallelism of SEBER (1976), was used to verify the heterogeneousness of reply among tested cultivars. The number of replications was estimated through the described methodology for HATHEWAY (1961). It can be concluded that to cultivars studied the number of replications that confers an adequate accuracy is equal to seven and the optimum plot size is $3.96 m^{2}$.

Key words: optimum plot size, number of replications, soil and experimental material heterogeneity.

\section{INTRODUÇÃO}

Atualmente, a cultura da soja encontra-se em destaque no cenário nacional, devido à rentabilidade que está proporcionando ao produtor rural. Dentre os fatores responsáveis por esse fato, está o setor da pesquisa que apresenta inovações técnicas e científicas melhorando cada vez mais o desempenho da propriedade rural. Pesquisas de qualidade passam por diversas fases, dentre elas estão o planejamento, condução, análise e divulgação dos resultados. O planejamento experimental adequado é fundamental para que os resultados apresentem qualidade.

\footnotetext{
${ }^{1}$ Engenheiro Agrônomo MSc, Doutorando Programa de Pós-graduação (PPG) em Fitotecnia, Escola Superior de Agricultura Luiz de Queiroz (ESALQ) ,Universidade de São Paulo. E-mail: martin@esalq.usp.br. Autor para correspondência.

${ }^{2}$ Engenheiro Agrônomo, Doutor, Professor Adjunto, Departamento de Fitotecnia, Universidade Federal de Santa Maria (UFSM), 97119900, Santa Maria, RS. E-mail: marcelo@ccr.ufsm.br.

${ }^{3}$ Engenheiro Agrônomo, MSc, Doutourando, PPG, Ciência do Solo, Centro de Ciências Rurais (CCR), UFSM.

${ }^{4}$ Engenheiro Agrônomo, Doutor, Professor, Titular, Departamento de Fitotecnia, CCR, UFSM.

${ }^{5}$ Aluno do curso de Agronomia, Bolsista Conselho Nacional de Desenvolvimento Científico e Tecnológico (CNPq), CCR,UFSM.

${ }^{6}$ Aluno do curso de Agronomia. Bolsista Programa Especial de Treinamento (PET), CCR, UFSM.

${ }^{7}$ Aluno do curso de Agronomia, CCR, UFSM.

${ }^{8}$ Engenheiro Agrônomo, Doutor, Professor Adjunto Departamento de Fitotecnia, Universidade Federal de Pelotas (UFPel).
} 
Diversos são os fatores que influenciam a precisão experimental. Dentre eles, destacam-se as heterogeneidades do solo e do material experimental, as competições intraparcelar e interparcelar, amostragem na parcela, dentre outros (STORCK et al., 2000). Quanto ao fator heterogeneidade do solo e do material experimental, este pode ser controlado através do uso de parcelas experimentais de tamanhos tais que neutralizam os efeitos da heterogeneidade (DE LA LOMA, 1955; LE CLERG et al., 1962; GOMEZ \& GOMEZ, 1984, MARTIN, 2003). O fato de parcelas situadas lado a lado possuírem diferenças é atribuída à heterogeneidade do solo (DE LA LOMA, 1955; LE CLERG et al., 1962 e GOMEZ \& GOMEZ, 1984). Além disso, a heterogeneidade do material experimental, em conjunto com a heterogeneidade dos solos, influencia diretamente o número de repetições e no tamanho da parcela experimental utilizada (STEEL et al., 1997).

Para a cultura da soja, a maior parte das informações referentes ao planejamento experimental a ser utilizado são produzidas por pesquisas estrangeiras e, muitas vezes, estas servem de base para a execução de experimentos no Brasil. Alguns resultados produzidos são apresentados por ODLAND \& GARBER (1928), que determinaram que o melhor tamanho de parcela possui uma área de $4,88 \mathrm{~m}^{2}$, com sulcos distanciados de $0,76 \mathrm{~m}$. WEBER \& HORNER (1957) chegaram a resultados semelhantes $\left(4,50 \mathrm{~m}^{2}\right)$, ou seja, parcelas de 3,1 unidades básicas, de 8 por 2 plantas. Esses resultados foram inferiores aos resultados obtidos por BRIM \& MASON (1959), que apresentaram o tamanho ótimo de parcela para a cultura da soja de $7,81 \mathrm{~m}^{2}$ (3 x 8 plantas). Já PIGNATARO \& GONÇALVES (1972) obtiveram em um experimento de uniformidade conduzido no Rio Grande do Sul, que o melhor tamanho de parcela corresponde a $1,80 \mathrm{~m}^{2}$, que pode ser estendido até $3,6 \mathrm{~m}^{2}$, sem perda de precisão.

Desta forma, o objetivo deste trabalho foi verificar se diferentes cultivares de soja alteram a estimativa do tamanho ótimo de parcela e do número de repetições.

\section{MATERIAL E MÉTODOS}

O experimento foi conduzido na área do Departamento de Fitotecnia no Campus da Universidade Federal de Santa Maria, no município de Santa Maria - RS, região climática da Depressão Central, a uma altitude de $95 \mathrm{~m}$, latitude $29^{\circ} 42$ ' $24^{\prime \prime} \mathrm{S}$ e longitude $53^{\circ} 48^{\prime} 42^{\prime \prime} \mathrm{W}$. O clima da região, segundo a classificação de Köeppen (MORENO, 1961) é do tipo Cfa - temperado chuvoso, com chuvas bem distribuídas ao longo do ano e subtropical do ponto de vista térmico. $\mathrm{O}$ solo pertence à Unidade de Mapeamento São Pedro, sendo classificado no Sistema Brasileiro de Classificação de Solos (EMBRAPA, 1999) como Argissolo Vermelho Distrófico arênico.

A correção do solo e a adubação da área foram realizadas de acordo com os resultados da análise de solo, em concordância com as Recomendações de Adubação e Calagem para os Estados do Rio Grande do Sul e Santa Catarina (COMISSÃO DE FERTILIDADE DO SOLO RS/SC, 1995) para a cultura da soja, utilizando $4,8 \mathrm{t} \mathrm{ha}^{-1} \mathrm{de}$ calcário PRNT 100\%, aplicado quatro meses antes da semeadura e $350 \mathrm{~kg} \mathrm{ha}^{-1}$ da formulação 0-25-25 na semeadura.

As cultivares utilizadas foram a BRS 137 (ciclo semiprecoce) e Fepagro-RS 10 (ciclo tardio), as quais foram semeadas em 19 de dezembro de 2000 , na densidade de 400000 plantas ha ${ }^{-1}$. Foram executadas todas as práticas culturais recomendadas para obtenção do máximo controle de insetos, moléstias e plantas daninhas, garantindo que o experimento ocorresse sem nenhuma interferência desses fatores.

A unidade experimental (parcela) foi composta por 18 filas de 8 metros de comprimento, distanciados 0,44 metros entre si. Para as avaliações, as parcelas principais foram subdivididas em 144 unidades básicas de um metro linear. $\mathrm{O}$ experimento foi circundado por quatro metros de bordadura lateral.

Para cada unidade básica (um metro), foi avaliado o rendimento de grãos, a $13 \%$ de umidade (RG). O delineamento experimental utilizado foi de blocos ao acaso, três blocos, com amostragem nas parcelas, cujo modelo é definido por STORCK et al. (2000). As pressuposições do modelo matemático para a variável rendimento de grãos (aleatoriedade dos erros estimados, homogeneidade da variância dos erros estimados entre os genótipos, aditividade do modelo matemático e normalidade da distribuição dos erros estimados) foram verificadas conforme aplicações de MARQUES (1999).

Para cada parcela principal, foram planejados tipos de parcela de diferentes tamanhos. Cada tipo de parcela teve $\mathrm{X} 1$ unidades básicas de comprimento (colunas) e X2 unidades básicas de largura (filas), formadas pelo agrupamento de unidades contíguas, de modo que $\mathrm{X} 1 * \mathrm{X} 2$ correspondem a $\mathrm{X}$ (tamanho da parcela em número de unidades básicas). Desta maneira, o número de repetições de cada tipo de parcela ficou limitado pela área total da parcela principal. Com isto, 
estabeleceram-se os seguintes parâmetros: $\mathrm{N}$ = número de parcelas, com $\mathrm{X}$ unidades básicas (UB) de tamanho calculado por $\mathrm{N}=144 / \mathrm{X} ; \mathrm{M}(\mathrm{x})=$ média das parcelas com X UB de tamanho; $\mathrm{V}(\mathrm{x})$ = variância entre parcelas de X UB de tamanho; $\mathrm{VU}(\mathrm{x})$ = variância por UB, calculada entre as parcelas de $\mathrm{X} \mathrm{UB}=\mathrm{V}(\mathrm{x}) / \mathrm{X}^{2}$ e CV $(\mathrm{x})$ $=$ coeficiente de variação entre as parcelas de $\mathrm{X}$ unidades básicas de tamanho. Tendo por base esses parâmetros, foi estimado o índice de heterogeneidade do solo "b", segundo a relação empírica $\mathrm{VU}(\mathrm{x})=\mathrm{V} 1 / \mathrm{X}^{\mathrm{b}}$, de SMITH (1938) para o valor do rendimento de grãos, em que: $\mathrm{VU}(\mathrm{x})$ = variância do valor observado por UB, calculada entre as parcelas com X UB; V1 = variância dos valores de parcelas constituídas de uma UB; $\mathrm{X}=$ número de UB que compõem a parcela no i-ésimo tipo de parcela considerado, $\mathrm{i}=1,2,3, \ldots, 15 ; \mathrm{e} \mathrm{b}=$ índice de heterogeneidade do solo.

O valor de $b$ foi estimado como um coeficiente de regressão linear, através da logaritmização da função $\mathrm{VU}(\mathrm{x})=\mathrm{V} 1 / \mathrm{X}^{\mathrm{b}}$, ou seja: $\log \mathrm{VU}(\mathrm{x})=\log \mathrm{V} 1-\mathrm{b} \log \mathrm{X}$, cuja estimação foi ponderada pelos graus de liberdade, associados aos 15 tipos de parcelas (STEEL et al., 1997).

Sobre as estimativas dos índices de heterogeneidade do solo $(\hat{b})$ foi aplicado o teste de paralelismo (SEBER, 1976) entre repetições dos genótipos e entre os genótipos, para testar a hipótese de paralelismo das regressões $\left(H_{0}: b_{1}=b_{2}=\ldots=b_{k}\right)$. A estatística do teste é

$$
F_{\text {cal }}=\frac{\left(S Q E_{H 0}-\sum_{K=1}^{K} S Q E\right) /(K-1)}{\sum_{K=1}^{K} S Q E /(n-2 K)}
$$

onde $\mathrm{SQE}_{H O}$ é a soma de quadrados do erro conjunto; $\sum_{k=1}^{K} S Q E \quad$ é o somatório das somas de quadrados do erro de cada regressão, $\mathrm{k}=1,2,3 \ldots \mathrm{K}=$ é o número de regressões comparadas; $\mathrm{n}$ é o número total de unidades básicas. Quando $\mathrm{F}_{\text {cal }}$ é maior que o valor de

$F_{t a b}\left(\alpha ; G L_{S Q E} ; G L_{S Q E_{H} 0}\right)$ não existe paralelismo entre os coeficientes de heterogeneidade do solo.

O tamanho da parcela foi estimado através do método da máxima curvatura modificada proposto por LESSMAN \& ATKINS (1963). O ponto de máxima curvatura de $\mathrm{CV}=\mathrm{A} / \mathrm{X}^{\mathrm{B}}$ define o tamanho ótimo da parcela que é dado pela função:

$$
\log X_{\text {ótimo }}=\left(\frac{1}{2 B+2}\right) \log \left(\frac{A^{2} B^{2}(2 B+1)}{(B+2)}\right)
$$

$\mathrm{O}$ número de repetições foi determinado através do método de HATHEWAY (1961), cuja fórmula para estimar o número de repetições em um experimento é : $\quad r=\frac{2(t 1+t 2)^{2} A^{2}}{d^{2} X^{b}}$ onde: $\mathrm{r}=$ número de repetições requerido para detectar diferenças de $d$ unidades; $d=$ diferença verdadeira entre duas médias de tratamentos expressa em porcentagem da média; $\mathrm{A}=$ estimativa do coeficiente de variação em percentagem para uma unidade básica; $O$ coeficiente $B$ mede a associação entre o $C V(x)$ e o tamanho da parcela $\mathrm{X} ; \mathrm{t} 1 \mathrm{l}=$ valor da tabela de $\mathrm{t}$ para testes de significância (bilateral a 5\%), e t2 = valor da tabela de t correspondente a 2 (1-P), onde P é a probabilidade de se obter resultados significativos $(0,80) ; \mathrm{X}=$ tamanho ótimo da parcela; $\mathrm{e} \mathrm{b}=$ índice de heterogeneidade do solo.

\section{RESULTADOS E DISCUSSÃO}

Para a variável rendimento de grãos, ajustada a $13 \%$ de umidade, as pressuposições do modelo matemático não foram violadas. Desta forma, pode-se dizer que a qualidade dos experimentos foi garantida para os testes de hipóteses (STORCK et al., 2000). Na tabela 1, verifica-se que não houve efeito significativo de bloco e também para o efeito de cultivar. Quanto ao erro experimental este não foi significativo, indicando que os blocos são homogêneos e as variações existentes são em decorrência da variação amostral, além do número de repetições possuir sua importância reduzida em favor da intensidade de amostragem. O rendimento de grãos médio foi de $2862 \mathrm{~kg} \mathrm{ha}^{-1}$, sendo este superior à média do estado do Rio Grande do Sul na safra 2000/01 (2339kg ha-1) (INDICAÇÕES TÉCNICAS, 2001). O coeficiente de variação do erro experimental foi de 42,60\% sendo classificado por LÚCIO (1997), como muito alto para a cultura. Esse elevado coeficiente de

Tabela 1 - Análise da variância segundo o delineamento blocos ao acaso com amostragem na parcela, fonte de variação (FV), graus de liberdade (GL), quadrados médios e médias por genótipo na variável rendimento de grãos de soja (RG); média e coeficiente de variação do erro experimental (CVe) e coeficiente de variação do erro amostral (CVea). Santa Maria - RS, 2001.

\begin{tabular}{lcc}
\hline FV & GL & RG $\left(\mathrm{t} \mathrm{ha}^{-1}\right)$ \\
\hline Bloco & 2 & $0,702^{\mathrm{ns}}$ \\
Genótipo & 1 & $0,905^{\mathrm{ns}}$ \\
Erro experimental & 2 & $3,392^{\mathrm{ns}}$ \\
Erro amostral & 858 & 1,488 \\
\hline BRS 137 & --- & $2,895^{\mathrm{ns}}$ \\
RS 10 & --- & 2,830 \\
\hline Média & --- & 2,862 \\
CVe & --- & 42,60 \\
CVea & --- & 64,35 \\
\hline
\end{tabular}

${ }^{\mathrm{ns}}=$ não significativo pelo teste $\mathrm{F}$, a $5 \%$ de probabilidade do erro.

Ciência Rural, v.35, n.2, mar-abr, 2005. 
Tabela 2 - Estimativas dos parâmetros da relação $\mathrm{CV}(\mathrm{x})=\mathrm{A} / \mathrm{X}^{\mathrm{B}}$, coeficiente de determinação $\left(\mathrm{r}^{2}\right)$, índice de heterogeneidade do solo $(\hat{b})$, estimativa do tamanho ótimo de parcela através do método de máxima curvatura modificada (MMCM), para cada um dos blocos, média $(\bar{X})$ e valores máximos dos parâmetros $\left(M_{x}\right)$, por cultivar e no global $(M G)$ e máximo global $\left(M_{x} G\right)$, com os respectivos números de repetições para a diferença verdadeira entre duas médias de tratamentos expressa em percentagem da média (d\%). Santa Maria - RS, 2001.

\begin{tabular}{|c|c|c|c|c|c|c|c|c|c|c|}
\hline \multirow{2}{*}{ Cultivares } & \multirow{2}{*}{ Bloco } & \multirow{2}{*}{ A } & \multirow{2}{*}{ B } & \multirow{2}{*}{$r^{2}$} & \multirow{2}{*}{$\hat{b}$} & \multirow{2}{*}{ MMCM } & \multicolumn{4}{|c|}{$\mathrm{d} \%$} \\
\hline & & & & & & & 5 & 10 & 20 & 40 \\
\hline \multirow[t]{3}{*}{ BRS 137} & 1 & $62,99 *$ & $0,72 *$ & 0,89 & $1,44 *$ & 8,90 & --- & --- & --- & -- \\
\hline & 2 & $52,88^{*}$ & $0,50 *$ & 0,89 & $0,99 *$ & 8,24 & --- & --- & --- & - \\
\hline & 3 & $46,71^{*}$ & $0,50 *$ & 0,94 & $1,01 *$ & 7,59 & --- & --- & --- & -- \\
\hline $\bar{X}$ & & 54,19 & --- & --- & 1,15 & 8,24 & 166 & 42 & 11 & 3 \\
\hline $\mathrm{M}_{\mathrm{x}}$ & & $62,99^{*}$ & --- & --- & --- & 8,90 & 109 & 28 & 7 & 2 \\
\hline \multirow[t]{3}{*}{ RS 10} & 1 & $46,81^{*}$ & $0,64 *$ & 0,89 & $1,28 *$ & 7,60 & --- & --- & --- & - \\
\hline & 2 & $43,08^{*}$ & $0,43^{*}$ & 0,94 & $0,85^{*}$ & 7,00 & --- & --- & --- & -- \\
\hline & 3 & $64,07^{*}$ & $0,62 *$ & 0,95 & $1,25^{*}$ & 9,24 & --- & --- & --- & -- \\
\hline $\bar{X}$ & & 51,32 & -- & --- & 1,13 & 7,95 & 161 & 41 & 11 & 3 \\
\hline $\mathrm{M}_{\mathrm{x}}$ & & 64,07 & --- & --- & --- & 9,24 & 151 & 38 & 10 & 3 \\
\hline $\mathrm{MG}$ & & $52,14 *$ & --- & --- & 1,14 & 8,18 & 158 & 40 & 10 & 3 \\
\hline$M_{x} G$ & & $64,07^{*}$ & --- & --- & 1,44 & 8,99 & 111 & 28 & 7 & 2 \\
\hline
\end{tabular}

* significativo pelo teste $\mathrm{F}$ a $5 \%$ de probabilidade de erro.

variação, tanto do erro experimental quanto o do erro amostral pode ser atribuído à elevada heterogeneidade do solo entre as parcelas vizinhas (Tabela 2).

A tabela 2 apresenta a estimativa dos parâmetros da relação $\mathrm{CV}(\mathrm{x})=\mathrm{A} / \mathrm{X}^{\mathrm{B}}$, onde todos apresentaram significância a $1 \%$ de probabilidade de erro, indicando desta forma, que os resultados possuem alta confiabilidade. O coeficiente de determinação apresenta valores superiores a 0,89 , o que indica que os dados possuem um bom ajustamento à equação. $\mathrm{O}$ índice de heterogeneidade do solo variou de 0,85 a 1,44 , o que significa grande heterogeneidade do solo, ou baixa correlação entre as parcelas adjacentes, indicando que as parcelas experimentais, neste local, devem ser maiores para evitar que um tratamento localize-se em uma região muito diferente da outra. $\mathrm{O}$ índice de heterogeneidade médio foi de 1,14, indicando que as parcelas vizinhas não são fortemente correlacionadas, fazendo com que as parcelas experimentais devam ser maiores para que elas não se localizem em solos muito contrastantes.

A estimativa do tamanho ótimo de parcela resultou em valores bastante próximos para as duas cultivares estudadas. A cultivar BRS 137 obteve como média, para o melhor tamanho de parcela o valor de 8,24 unidades básicas $\left(3,62 \mathrm{~m}^{2}\right)$ e a cultivar RS 10 obteve o valor médio de 7,95 unidades básicas $\left(3,50 \mathrm{~m}^{2}\right)$. A partir destes valores médios, pode-se verificar que as cultivares possuem desempenhos semelhantes quanto aos tamanhos de parcela obtidos. Contudo, seria mais indicado a utilização do valor máximo de cada um dos parâmetros para, desta forma, obter-se uma proteção estatística aos resultados. Sendo assim, os valores de 8,90 UB e 9,24 UB são indicados para as cultivares BRS 137 e RS 10, respectivamente. O valor máximo global para as cultivares de soja foi de $8,99 \mathrm{UB}\left(3,96 \mathrm{~m}^{2}\right)$, que é um pouco inferior ao valor encontrado para o tamanho de parcela máximo da cultivar RS 10, devido à combinação dos parâmetros máximos utilizados produzirem resultados diferentes, quando submetidos ao cálculo do tamanho ótimo de parcela.

A uniformização dos ensaios de indicação de cultivares de soja poderia ser realizada com parcelas de $3,96 \mathrm{~m}^{2}$, permitindo maior precisão experimental. Esse valor é superior aos valores encontrados por PIGNATARO \& GONÇALVES (1972), que obtiveram o melhor tamanho de parcela correspondente a $1,80 \mathrm{~m}^{2}$.

A partir do número de repetições (Tabela 2 ), verifica-se que ao se comparar as cultivares utilizadas (número médio de repetições), que este apresentou variação quase que desprezível. Desta forma, o número de repetições, assim como o tamanho ótimo de parcelas pode ser o mesmo para as duas cultivares em estudo. A maior variação do número de repetições obtida para as duas cultivares deu-se ao utilizarem-se os valores máximos dos parâmetros que compõem o cálculo. No entanto, à medida que se aumenta a diferença verdadeira entre duas médias estes valores tendem à aproximação. Quando os

Ciência Rural, v.35, n.2, mar-abr, 2005. 
Tabela 3 - Valores da estatística $\mathrm{F}$ calculado $\left(\mathrm{F}_{\mathrm{cal}}\right)$ para o teste de paralelismo dos índices de heterogeneidade do solo ( $\hat{b}$ ), entre os blocos da mesma cultivar e entre as cultivares, graus de liberdade do resíduo (GLr) e somatório de quadrados dos resíduos (SQr). Santa Maria - RS, 2001.

\begin{tabular}{|c|c|c|c|c|}
\hline Classe & GLr & SQr & $\hat{b}$ & $\mathrm{~F}_{\mathrm{cal}}$ \\
\hline \multirow[t]{3}{*}{ BRS 137} & 13 & 0,04399789 & $1,44^{*}$ & \\
\hline & 13 & 0,19059602 & $0,99 *$ & \\
\hline & 13 & 0,0953901 & $1,01^{*}$ & \\
\hline Soma & 39 & 0,32998401 & & \\
\hline Geral & 44 & 3,70391479 & & $194,27 *$ \\
\hline \multirow[t]{3}{*}{ RS 10} & 13 & 0,6618297 & $1,28^{*}$ & \\
\hline & 13 & 0,01200558 & $0,85^{*}$ & \\
\hline & 13 & 0,02189292 & $1,25^{*}$ & \\
\hline Soma & 39 & 0,10008147 & & \\
\hline Geral & 44 & 2,70213523 & & $329,32^{*}$ \\
\hline BRS 137 & 43 & 3,70391479 & $1,15^{*}$ & \\
\hline RS 10 & 43 & 2,70213523 & $1,04 *$ & \\
\hline Soma & 86 & 6,40605002 & & \\
\hline Geral & 89 & 6,45628799 & & $0,32^{\mathrm{ns}}$ \\
\hline
\end{tabular}

$* \mathrm{e}^{\text {ns }}$ : Significativo e não-significativo pelo teste F, em nível de $5 \%$ de probabilidade de erro, respectivamente.

valores da diferença verdadeira entre duas médias é de $20 \%$, o número de repetições obtido foi de 7 , mas, quando a diferença verdadeira for inferior a este valor, as aplicações práticas dos resultados ficam prejudicadas devido ao elevado custo tanto financeiro como de mão-de-obra para a condução destes ensaios.

A tabela 3 apresenta o teste de paralelismo entre os blocos dentro da mesma cultivar e entre as cultivares estudadas. Percebese que não existe paralelismo de resposta entre os blocos das cultivares estudadas, indicando que a heterogeneidade do solo é responsável por parcelas situadas lado a lado possuírem variações, esses resultados concordam com DE LA LOMA, 1955; LE CLERG et al., 1962 e GOMEZ \& GOMEZ, 1984. Quanto ao teste de paralelismo entre as cultivares estudadas, verifica-se que os índices de heterogeneidade do solo possuem comportamento paralelo, indicando que as cultivares estudadas não são heterogêneas, fazendo com que desta forma, seja permitido o uso do mesmo tamanho de parcela para as duas cultivares. Utilizando-se da mesma metodologia, MARTIN (2003) verificou que para híbridos de milho, somente era possível a utilização do mesmo tamanho de parcela, quando se utilizava o máximo valor global obtido para as quatro bases genéticas estudadas.

\section{CONCLUSÕES}

Para as cultivares estudadas o número de repetições que confere uma precisão adequada é igual a sete e o tamanho ótimo de parcela é de $3,96 \mathrm{~m}^{2}$.

\section{REFERÊNCIAS BIBLIOGRÁFICAS}

BRIM, C.A.; MASON, D.D. Estimates of optimum plot size for soybean yield trial. Agronomy Journal, Madison, v.51, p.331$334,1959$.

COMISSÃO DE FERTILIDADE DO SOLO-RS/SC. Recomendações de adubação e calagem para o estado do Rio Grande do Sul e Santa Catarina. 3.ed. Passo Fundo : SBCC - Núcleo Regional Sul, EMBRAPA/CNPT, 1995. 223p.

DE LA LOMA, J.L. Experimentación agrícola. México: Hispano Americana, 1955. 500p.

EMBRAPA. Centro Nacional de Pesquisa de Solos (Rio de Janeiro, RJ). Sistema brasileiro de classificação dos solos. Brasília : Embrapa-SPI, 1999. 412p.

GOMEZ, K.A.; GOMEZ, A.A. Statistical procedures for agricultural research. 2.ed. New York : John Wiley, 1984. 680p.

HATHEWAY, W.H. Convenient plot size. Agronomy Journal, Madison, v.53, n.4, p.279-280, 1961.

INDICAÇÕES TÉCNICAS. XXIX Reunião de Pesquisa de Soja da Região Sul (29.: 2001: Porto Alegre). Indicações técnicas para a cultura da soja no Rio Grande do Sul e Santa Catarina 2001/ 2002. Porto Alegre: FEPAGRO, 2001. 138p.

Ciência Rural, v.35, n.2, mar-abr, 2005. 
LE CLERG, E.L. et al. Field plot technique. Minneapolis : Burgees, 1962. 373p.

LESSMAN, K.J.; ATKINS, R.E. Optimum plot size and relative efficiency of lattice designs for grain sorghum yield test. Crop Science, Madison, v.3, p.477-481, 1963.

LÚCIO, A.D. Parâmetros da precisão experimental das principais culturas anuais do Estado do Rio Grande do Sul. 1997. 64f. Dissertação (Mestrado em Agronomia) - Curso de Pósgraduação em Agronomia, Universidade Federal de Santa Maria.

MARQUES, D.G. As pressuposições e a precisão dos ensaios de competição de cultivares de milho no estado do Rio Grande do Sul. 1999. 42f. Dissertação (Mestrado em Agronomia) Curso de Pós-graduação em Agronomia, Universidade Federal de Santa Maria.

MARTIN, T.N. Contribuição das bases genéticas de milho para o plano experimental. 2003. 89f. Dissertação (Mestrado em Agronomia) - Curso de Pós-graduação em Agronomia, Universidade Federal de Santa Maria.

MORENO, J.A. Clima do Rio Grande do Sul. Porto Alegre: Secretaria da Agricultura, 1961. 41p.
ODLAND, T.E.; GARBER, R.J. Size of plot and number of replication in field experiments with soybeans. Journal of the American Society of Agronomy, v.20, p.93-108, 1928.

PIGNATARO, I.A.B.; GONÇALVES, H.M. Estimativa de melhor tamanho de parcela para experimentos de soja. Agronomia Sulriograndense, v.8, n.2, p.153-159, 1972.

SEBER, G.A.F. Linear regression analysis. NewYork : John Wiley, 1976. 465p.

SMITH, H.F. An empirical law describing heterogeneity in the yields of agricultural crops. Journal of Agricultural Science, Cambridge, v.28, p.1-23, 1938.

STEEL, R.G.D. et al. Principles and procedures of statistics. 3.ed. New York: McGraw Hill, 1997. 666p.

STORCK, L. et al. Experimentação vegetal. Santa Maria : UFSM, 2000. 198p.

WEBER, C.R.; HORNER, T.W. Estimates of cost and optimum plot size and shape for measuring yield and chemical characters in soybeans. Agronomy Journal, Madison, v.49, p.444-449, 1957. 